Correcting for solvent-solvent electrostatic cutoffs considerably improves the ion-pair potential of mean force

Haluk Resat

Citation: The Journal of Chemical Physics 110, 6887 (1999); doi: 10.1063/1.478801

View online: http://dx.doi.org/10.1063/1.478801

View Table of Contents: http://aip.scitation.org/toc/jcp/110/14

Published by the American Institute of Physics

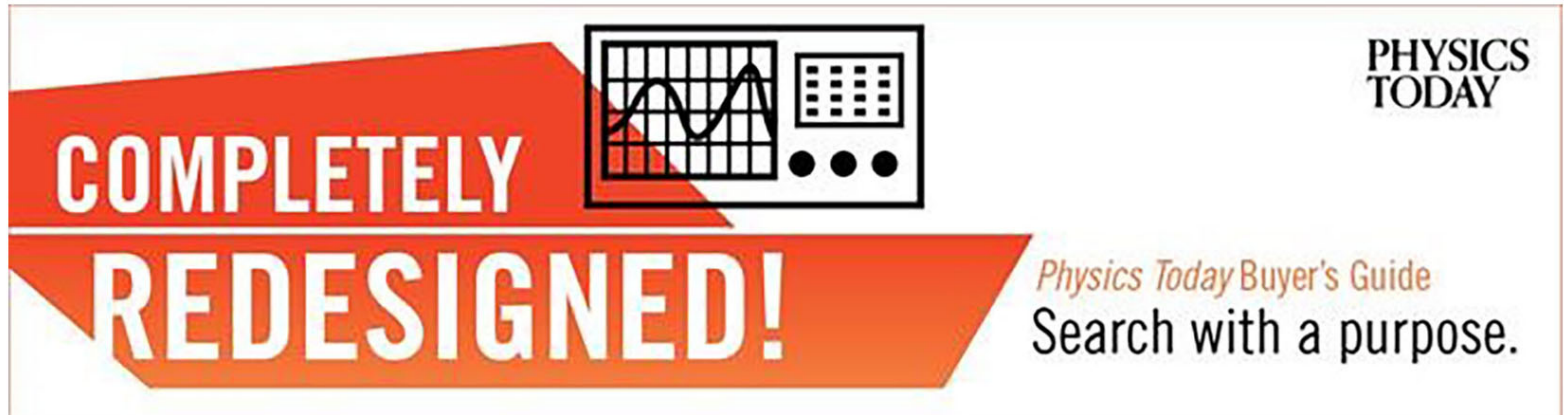




\title{
Correcting for solvent-solvent electrostatic cutoffs considerably improves the ion-pair potential of mean force
}

\author{
Haluk Resat ${ }^{\text {a) }}$ \\ Department of Physics, Faculty of Arts and Sciences, Koç University, Istinye, Istanbul, Turkey
}

(Received 3 September 1998; accepted 11 January 1999)

\begin{abstract}
A recently developed algorithm based on the continuum treatment of the solvent molecules beyond the electrostatic cutoff sphere is applied to the potential of mean force results between sodium and chloride ions to study the effects of the solute-solvent and solvent-solvent cutoff errors. The results show that although the solute-solvent correction improves the thermodynamic results slightly, physically realistic results are obtained only when the solvent-solvent correction is applied. This further supports past findings that proper treatment of solvent-solvent interactions is as important as that of the solute interactions, and should not be ignored. (C) 1999 American Institute of Physics. [S0021-9606(99)51214-2]
\end{abstract}

\section{OVERVIEW}

The numerical calculation of the thermodynamic properties of solvated systems has been proven to depend on the methods employed. One particular aspect which gives rise to problems of inconsistency in molecular simulations is the inadequate treatment of electrostatic cutoffs. This problem has attracted the attention of many groups in recent years. An extensive listing of such studies may be found in the references listed in our earlier work, ${ }^{1,2}$ or in a recent study by Hünenberger and van Gunsteren. ${ }^{3}$

Although the Ewald sum method avoids cutoffs and seems to perform well, it can have severe artifacts and is not that easy to implement. ${ }^{3}$ In comparison to the Ewald method, the spherical cutoff method is a conceptually simpler and a less expensive alternative whose implementation is much easier. However, the employed spherical cutoffs introduce errors into the calculated molecular and thermodynamic properties. To improve the quality of the thermodynamic properties computed using the spherical cutoff method, we have recently developed an algorithm ${ }^{1,2}$ which allows for the correction of the errors introduced by the employed spherical cutoffs. The method was successfully applied to correct for the solute-solvent ${ }^{1}$ and the solvent-solvent ${ }^{2}$ cutoffs.

\section{METHOD}

Since it has been extensively detailed in Refs. 1 and 2, only a summary of the cutoff correction algorithm will be given here. The algorithm to calculate the free energy differences consists of three steps: (a) Performing the desired free energy simulation, the sodium-chloride potential of mean force in this case. The configurations generated during the performed molecular simulation are then used to compute (b) the solute-solvent cutoff correction, ${ }^{1}$ and (c) the solventsolvent cutoff correction. ${ }^{2}$

In calculating the solute-solvent cutoff correction, the Poisson equation

$$
\nabla \cdot \epsilon(r) \nabla \Psi(r)+4 \pi q \rho^{f}(r) / k_{B} T=0,
$$

is solved for the electrostatic potential $\Psi(r)$. In Eq. (1), $\Psi(r)$ is expressed in units of $k_{B} T$, and $\rho^{f}(r)$ is the fixed charge distribution. The dielectric function $\epsilon(r)$ defines the polarizability of the system. In this work the Poisson equation is solved by placing the complex formed by the solute and all the solvent molecules within the solute's interaction volume into a dielectric continuum and evaluating the neglected solvation free energy. Once the reaction potentials $\Phi(r)$ at the interaction sites are found, then the solutesolvent cutoff correction is calculated by performing the sum over the interaction sites of the solute molecules ${ }^{1}$

$$
G=\frac{1}{2} \sum_{k} q_{k} \Phi_{k}
$$

The solvent-solvent cutoff correction can be calculated similarly using the Poisson equation for the solvation shell of each solvent molecule. As discussed in Ref. 2 this would, however, be quite expensive because molecular simulations usually employ thousands of solvent molecules; solving the Poisson equation once for each solvent molecule would be computationally demanding. To save expense, a simpler approach utilizing the analytical reaction field formalism can be employed, and it was shown to be a viable alternative. ${ }^{1,2}$

Similar to the Poisson equation calculations, in the reaction field method one creates an interaction sphere for each solvent molecule and calculates the reaction field of the surrounding solvent (i.e., that of the neglected, extending to infinity part of the solvent) analytically. The solvent-solvent cutoff correction is then computed using the sum given in Eq. (2) where this time the reaction potentials $\Phi(r)$ are determined using the analytical reaction field expression ${ }^{2}$

$\Phi_{k}=\sum_{n=0}^{\infty} \frac{(n+1)\left(1-\epsilon_{r}\right)}{(n+1) \epsilon_{r}+n} \frac{r_{k}^{n}}{R_{c}^{2 n+1}} \sum_{l=1} q_{l} r_{l}^{n} P_{n}\left(\cos \theta_{k l}\right)$,

where the $l$ summation is over all the charge sites within the cavity. 


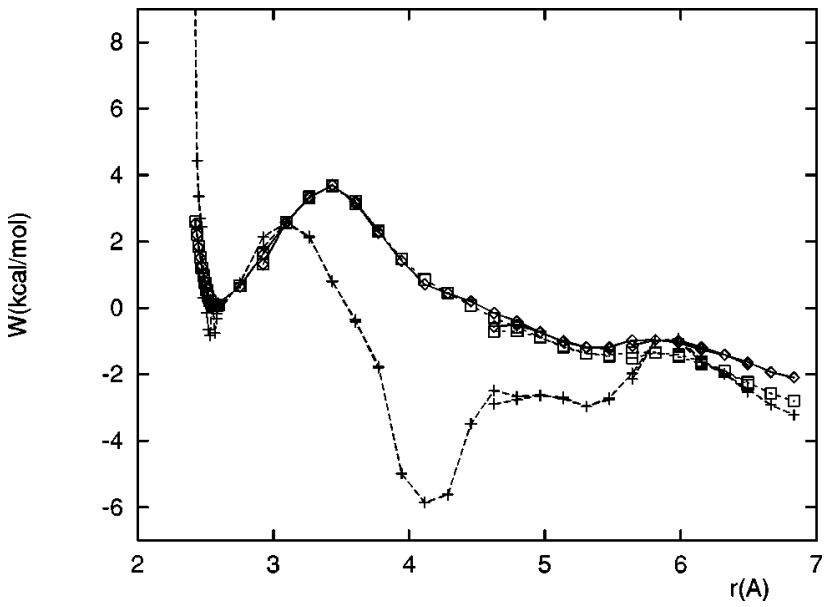

FIG. 1. Potential of mean force between sodium and chloride ions. The distance $r$ is in $\AA$ and the free energy is in $\mathrm{kcal} / \mathrm{mol}$. Line with squares: Pmf without any correction; with diamonds: pmf with only the solute-solvent correction; and with pluses: pmf with the solute-solvent and the solventsolvent cutoff corrections.

\section{RESULTS AND DISCUSSION}

The cutoff errors, in principal, are expected to be more significant for highly polar systems. For this reason, to severely test our algorithm, it is here applied to compute the spherical cutoff corrections for a highly polar system. As the test case, the distance dependent free energy (the potential of mean force) between sodium and chloride ions in aqueous solution was chosen. In an earlier study the potential of mean force (pmf) between sodium and chloride ions was computed. ${ }^{4}$ The computed pmf, Fig. 1, was not in accord with physical expectations in two major ways: (i) It did not reproduce the solvent separated configuration as the most stable minimum, and (ii) the large separation distance behavior contradicted the expectations by not leveling off (Fig. 1). Since the simulations were well converged, it was speculated that the observed unphysical results were most likely due to the employed cutoffs. ${ }^{4}$ Results of this earlier study established that the $\mathrm{Na}-\mathrm{Cl}$ system would be a good case to severely test our cutoff algorithm. The present study confirms that the cause of the unphysical results were indeed due to the employed cutoffs. The corrected results contain all the physical features that a proper $\mathrm{Na}-\mathrm{Cl}$ pmf is expected to have.

The pmf between sodium and chloride was calculated in Ref. 4 using a Monte Carlo simulation in which the molecular interactions were truncated. In the simulation, the $\mathrm{Na}-\mathrm{Cl}$ pair was treated as a group and the ion pair was assumed to interact with all the waters inside the unit cell (minimum image cutoff). The simulation employed periodic boundary conditions and the minimum image rule was used to determine which water (among its replicated copies) was closest to the center of mass of the ion pair. The grand canonical ensemble was used and there were 565 water molecules on average in the unit cell with dimensions $35.15 \AA \times 22 \AA \times 22$ $\AA$. The solvent-solvent interactions were truncated using a spherical cutoff at $11 \AA$. To achieve consistency, cutoff correction calculation conditions were matched with the molecular simulation setup. The solute-solvent cutoff correction $^{1}$ was calculated by solving the Poisson equation for which the dielectric constant inside the unit cell was set to be one, and the solvating dielectric continuum outside the cell, had a dielectric constant of 78. The site charges of the ions and the waters inside the unit cell formed the charges appearing in the Poisson equation.

The solvent-solvent cutoff correction ${ }^{2}$ was calculated using the generalized reaction field method. An interaction sphere complex was formed for each solvent molecule and the reaction field energies were calculated for this collection of charges inside the sphere. Since each water either interacts with both ions at the same time or not, the reaction field contribution when the ions are outside the interaction sphere of a water molecule was assumed to vanish. The ions were included in the reaction field calculations when they were inside the water's interaction sphere. Further details of the pmf molecular simulation and the cutoff correction calculations may be found in Ref. 4 and Refs. 1 and 2, respectively.

The pmf between the sodium and the chloride ion is believed to have several minima. Judging from the solubility properties of $\mathrm{NaCl}$, in dilute solutions the contact ion pair (CIP) is less stable than that of the solvent separated ion pair (SSIP) where the ions are not in direct contact but there are bridging water molecules in between. However, the computed locations of the free energy minima and the height and the positions of the barriers separating these minima show significant differences among various studies. ${ }^{5-10}$ To make comparison with earlier studies, one can use the solubility properties and the potential of mean force results calculated using the Ewald sum. ${ }^{5-10}$ With these as the criteria, it can be said that a reasonable pmf curve is expected have a CIP minimum in the 2.5-3.0 $\AA$ range, another minimum (SSIP) at $\sim 2 \AA$ larger separation, and several other minima at even larger distances. Since low concentration $\mathrm{NaCl}$ salt easily dissolves in water, the dissociation barrier separating the CIP and SSIP states should not be too large (e.g., $1-4 \mathrm{kcal} / \mathrm{mol}$ at room temperature). Since the solvent bridged ion pair is favored, its free energy should be lower than that of the contact ion pair configuration.

The uncorrected and cutoff corrected results for the potential of mean force between sodium and chloride ions are shown in Fig. 1. Even though the solute-solvent cutoff correction improves the pmf results, the improvement is only marginal. The solute-solvent correction is a smooth function of the distance (i.e., the dipole moment) between the ions. It improves the decay in the results by about $1 \mathrm{kcal} / \mathrm{mol}$ without effecting the features in the function. Note that the total solute charge is zero regardless of the ion separation distance, therefore, the solute-solvent correction is mainly that of a large dipole enclosed in a cavity. In contrast to the solute cutoff, the solvent-solvent cutoff correction causes a major change in the results, both qualitatively and quantitatively. The solvent-solvent cutoff correction is much larger in this case, on the order of $6 \mathrm{kcal} / \mathrm{mol}$ at certain distances. One reason for such a large solvent-solvent cutoff correction is that there are many water molecules in the unit cell and even a small solvent-solvent cutoff correction for each water molecule can add up to give a large cumulative solvent-solvent cutoff correction. The fact that the solvent-solvent cutoff 
correction can be quite large (even up to tens of $\mathrm{kcal} / \mathrm{mol}$ ) has been observed before. ${ }^{2,11}$ An interesting finding is that solvent cutoff corrections make major qualitative changes in the pmf at distances where the ion pair forms a solvent bridged configuration. This is probably due to the unique patterns that the water molecules adapt when the ions are separated just enough to squeeze in bridging waters.

The pmf results with both the solute and the solvent cutoff corrections has the features expected from a physical $\mathrm{Na}-\mathrm{Cl}$ pmf: The solvent separated minimum at $4.2 \AA$ is about $5 \mathrm{kcal} / \mathrm{mol}$ lower than the contact ion pair at $2.6 \AA$. There is also a second solvent bridged configuration at $5.3 \AA$ which is also more stable than the contact pair. The dissociation barrier separating the ion contact pair from the solvent separated pair is $\sim 3.4 \mathrm{kcal} / \mathrm{mol}$, in accord with the earlier studies. It should be noted again that there are significant differences among various earlier studies ${ }^{5-10}$ due to the utilized method differences, and more importantly due to the employed interaction potential models, both for the ions and the waters. Therefore, a direct comparison of our results with earlier studies is not straightforward and was avoided.

Based on the results reported here, and on the earlier studies, ${ }^{1,2}$ it can be confidently stated that our correction algorithm performs very well. In the test case studied here our method was applied to correct a pmf which had unrealistic features. Such features were possibly due to the artifacts of the imposed truncations during the molecular simulation computations. The cutoff correction method appropriately eliminated the unphysical features in the investigated pmf, and introduced features which were expected based on solubility properties.

As a final note, the cutoff algorithm developed in Refs. 1 and 2 and tested here has the major advantages that it is conceptually simple and very easy to implement, and it only requires a small increase in the computational time.

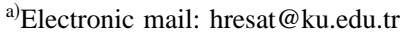

${ }^{1}$ H. Resat and J. A. McCammon, J. Chem. Phys. 104, 7645 (1996)

${ }^{2}$ H. Resat and J. A. McCammon, J. Chem. Phys. 108, 9617 (1998).

${ }^{3}$ P. H. Hünenberger and W. F. van Gunsteren, J. Chem. Phys. 108, 6117 (1998).

${ }^{4}$ H. Resat, M. Mezei, and J. A. McCammon, J. Phys. Chem. 100, 1426 (1996).

${ }^{5}$ L. X. Dang, J. E. Rice, and P. A. Kollman, J. Chem. Phys. 93, 7528 (1990).

${ }^{6}$ E. Guardia, R. Rey, and J. A. Padro, Chem. Phys. 155, 187 (1991).

${ }^{7}$ R. Rey and E. Guardia, J. Phys. Chem. 96, 4713 (1992).

${ }^{8}$ D. E. Smith and A. D. J. Haymet, J. Chem. Phys. 96, 8450 (1992).

${ }^{9}$ G. Hummer, D. M. Soumpasis, and M. Neumann, Mol. Phys. 81, 1155 (1993).

${ }^{10}$ D. E. Smith and L. X. Dang, J. Chem. Phys. 100, 3757 (1994).

${ }^{11}$ R. H. Wood, J. Chem. Phys. 103, 6177 (1995). 